\title{
Randomized clinical trial of tetracycline hydrochloride bandage and paste treatments for resolution of lesions and pain associated with digital dermatitis in dairy cattle
}

\author{
J. H. Higginson Cutler, ${ }^{* 1}$ G. Cramer, ${ }^{\star} \dagger$ J. J. Walter,ł S. T. Millman,§ and D. F. Kelton* \\ *Department of Population Medicine, University of Guelph, Guelph, ON, Canada N1G 4W1 \\ †College of Veterinary Medicine, University of Minnesota, St. Paul 55108 \\ fDepartment of Companion Animals, University of Prince Edward Island, Charlottetown, PEI, Canada C1A 4P3 \\ §Veterinary Diagnostic and Production Animal Medicine, lowa State University, Ames 50011
}

\begin{abstract}
Digital dermatitis is an infectious disease that causes lameness in dairy cattle, a primary welfare concern of the dairy industry. One of the common treatments for this painful hoof disease is through the application of an antibiotic bandage that must be removed following treatment. The objectives of this randomized clinical trial were to determine if topical application of tetracycline hydrochloride in a paste would be as therapeutically effective for the treatment of digital dermatitis as a powdered form of tetracycline hydrochloride held in place by a bandage, and to quantify pain associated with digital dermatitis lesions. Two hundred and fourteen Holstein cow hooves with digital dermatitis lesions were randomly assigned to 1 of 3 treatments: a tetracycline hydrochloride paste, tetracycline hydrochloride powder held in place with a bandage for $2 \mathrm{~d}$, or a negative (untreated) control. Lesions were examined at 2 time periods: 3 to $7 \mathrm{~d}$ posttreatment and 8 to $12 \mathrm{~d}$ posttreatment to determine healing rates. Nociceptive thresholds were measured using a pressure algometer to quantify the pain at the lesion site. The tetracycline hydrochloride paste was as effective as the powdered bandage treatment in terms of healing rates, with 47.4 and $57.1 \%$ hooves healed at 8 to $12 \mathrm{~d}$ posttreatment, respectively. Both treatments were more effective than the control, in which no lesions healed 8 to $12 \mathrm{~d}$ following initial examination. Mean $( \pm \mathrm{SE})$ nociceptive thresholds for active, healing, and healed lesions differed, with limb-withdrawal response occurring at 7.45 $( \pm 0.67) \mathrm{kg}, 12.84( \pm 1.85) \mathrm{kg}$, and censored to $25 \mathrm{~kg}$ (maximum value of algometer) of force applied, respectively. However, active lesions were not consistently associated with pain, as maximum force was tolerated when applied to $19 \%$ of active lesions, perhaps due to variability in stoicism between individual cattle or due
\end{abstract}

Received November 15, 2012.

Accepted June 27, 2013.

${ }^{1}$ Corresponding author: jhiggins@uoguelph.ca to changes in pain during the progression of infection. In conclusion, tetracycline hydrochloride paste was as effective as tetracycline hydrochloride bandage, eliminating the need for bandage removal following treatment application. Digital lesions can be painful during both active and healing stages, suggesting the need for treatment and husbandry interventions for pain mitigation.

Key words: digital dermatitis, lameness, tetracycline hydrochloride, pressure algometry

\section{INTRODUCTION}

Lameness is considered one of the most important animal welfare concerns for the dairy industry. This is due to many factors, including pain, effect on performance, and high prevalence in the industry. Digital dermatitis is an infectious disease that commonly causes lameness in dairy cattle. Since first being described in Italy by Cheli and Mortellaro (1974), digital dermatitis has been a persistent problem.

Prevalence of digital dermatitis remains high in different countries. In a Canadian cross-sectional study including 204 Ontario dairy farms, $69.7 \%$ of tie-stall herds and $92.1 \%$ of freestall herds had animals with digital dermatitis (Cramer et al., 2008). In the United States, earlier estimates of prevalence showed that $43.5 \%$ of dairy herds had digital dermatitis (Wells et al., 1999). Additionally, those authors determined that $81.9 \%$ of cows with digital dermatitis lesions were lame (Wells et al., 1999), suggesting that this is a painful condition in at least some animals.

As reviewed by Laven and Logue (2006), treatment for digital dermatitis usually occurs at 1 of 2 levels: the herd level in the form of footbaths or at the individual cow level in the form of systemic antibiotic administration or application of topical antibiotic or disinfectant at the lesion site. Antibiotic footbaths appear to provide reasonable herd-level prevention as well as some treatment of lesions (Laven and Proven, 2000). Those 
authors found footbaths containing erythromycin significantly reduced lameness within cow by $41 \%$ and pain by $55 \% 4 \mathrm{~d}$ after treatment compared with untreated control cows, although indicators of pain were not defined. Due to concerns with the use of antibiotics, footbathing with alternative nonantibiotic solutions for prevention of digital dermatitis rather than treatment are becoming more common.

Systemic antibiotic treatment of lameness is generally believed to be ineffective (Blowey and Sharp, 1988), although little scientific literature is available on the subject. Treatment of digital dermatitis with an antibiotic spray has been shown to be effective, whereas sprays containing antibiotic alternatives are not as effective as topical treatments for digital dermatitis (reviewed in Laven and Logue, 2006). Topical treatments with an antibiotic bandage have been used to treat lesions at the cow level (Read and Walker, 1998; Cramer et al., 2008; Berry et al., 2010), and are commonly used in North America. However, if the bandage is not removed, this could cause damage to the leg of the cow. Anecdotal reports of injuries to cows from lack of bandage removal are numerous; however, this finding has not been reported in published research papers. A case series from companion animal veterinary medicine found that ischemic injuries from bandages were due to direct pressure necrosis or ischemic necrosis (Anderson and White, 2000), both of which are assumed to be painful conditions. The use of an antibiotic paste for digital dermatitis treatment would eliminate the need to place bandages on lesions, thus removing the chance of damage from lack of removal.

Whereas many studies have assessed the effectiveness of treatments through clinical cure, little attention has been paid on the effectiveness of such treatments to reduce pain associated with lesions. Lameness pain is particularly important for animal welfare, as cattle stimulate pain responses during activities that require them to be upright, such as during feeding and milking. To avoid pain, lame cattle spend more time lying and less time feeding (Galindo and Broom, 2002), which is likely to affect performance. Quantification of pain associated with digital dermatitis and throughout the healing process is needed to identify periods during which pain control or other interventions would be beneficial for the welfare and productivity of the cow. Previously, researchers subjectively scored pain associated with digital dermatitis using a 3-point scale based on reaction to water sprayed at the lesion (Hernandez et al., 1999), and determined that these pain scores decreased in cattle treated with oxytetracycline or a commercial copper formulation.

The primary objective of this randomized clinical trial was to determine if topical application of tetracycline hydrochloride in a paste would be as therapeutically effective for treatment of digital dermatitis lesions as tetracycline hydrochloride held in place by a bandage. A second objective was to quantify pain associated with digital dermatitis lesions. All procedures were approved by the University of Guelph Animal Care Committee (Guelph, ON, Canada; Animal Use Protocol 09R089).

\section{MATERIALS AND METHODS}

\section{Animals and Management}

This study was conducted at the University of Guelph Elora Dairy Research Station (Elora, ON, Canada). Holstein cows were housed in either tie-stall or freestall sections of the facility, with approximately $20 \%$ of the herd housed in the freestall portion of the barn. Throughout the trial period, a total of 234 cows were housed in the facility. All animals entered a single milking parlor twice daily for milking and were fed a TMR 3 times daily. As a preventive treatment for digital dermatitis, all animals on the farm, regardless of inclusion in the trial, walked through a footbath 3 times weekly upon exit from the parlor as per farm protocol (twice-weekly treatments of copper sulfate at $58 \mathrm{~g} / \mathrm{L}$ and once-weekly treatments of tetracycline at $1.16 \mathrm{~g} / \mathrm{L})$.

\section{Experimental Procedure and Treatments}

Cows had their hooves inspected while restrained in a hydraulic lift table approximately every $3 \mathrm{mo}$, with full hoof trimming occurring every 6 mo from June 2009 to August 2010. Digital dermatitis lesions were scored upon visual inspection by a trained veterinarian specializing in dairy lameness (author G. Cramer) and a researcher trained by the veterinarian to score lesions (author J. H. Higginson Cutler) during hoof inspection at 5 time periods: June 2009, September 2009, January 2010, May 2010, and August 2010, termed trim date. Throughout the trial, all digital dermatitis lesions were scored as active, healing, or healed by the authors ( $\mathrm{J}$. H. Higginson Cutler and G. Cramer) at the time of hoof examination. If manure presence prevented observation of lesions, this was removed with a towel. Active lesions were raw, moist lesions with tufted or granular surfaces (Hernandez and Shearer, 2000) and were typically pink-red in color. Lesions that were healing were dry and developing a scab (modified from Manske et al., 2002a,b). Healed lesions showed no visible sign of moist surface or scab. Anatomic location of lesions were also recorded on data sheets using the numbering system shown in Figure 1 and digital photographs of all lesions were taken. 


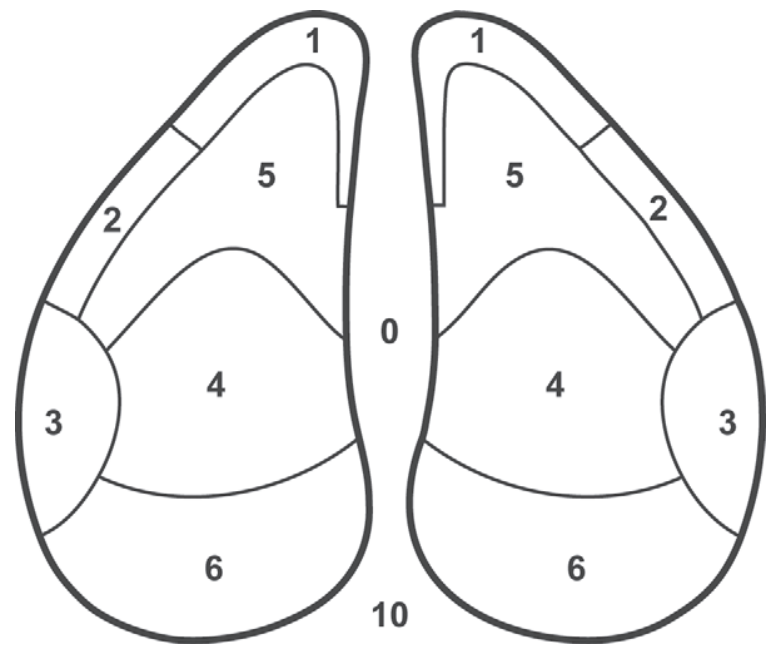

Figure 1. Lesion anatomic location numbering system. Used with permission (Shearer et al., 2004).

Hooves with active lesions were randomly assigned to 1 of 3 treatment groups. A randomization chart was prepared before the beginning of the study through random draw and lesions were assigned to treatment from the chart in order of appearance. Due to hoof-level assignment of treatment, 1 cow could be exposed to different treatments on other limbs and hooves could be included more than once in the trial if lesions appeared more than once throughout the trial period. The paste treatment consisted of $175 \mathrm{~mL}$ of propylene glycol, $175 \mathrm{~mL}$ of vinegar, and $150 \mathrm{~g}$ of tetracycline hydrochloride (Onycin 1000; Vétoquinol Canada Inc., Lavaltrie, QC, Canada), applied directly to the lesion with a paintbrush. This resulted in approximately 2 to $5 \mathrm{~g}$ of tetracycline applied per treatment. The bandage treatment consisted of 2 to $5 \mathrm{~g}$ of tetracycline hydrochloride (Onycin 1000; Vétoquinol Canada Inc.) covering a $5 \times 5$-cm square gauze and secured to the lesion using a bandage (Vetrap; 3M, St. Paul, MN). Bandages were removed from the hoof approximately $48 \mathrm{~h}$ after application. The negative control consisted of no therapeutic intervention. Severely lame cows, with little to no weight bearing on the affected limb, were assigned to the paste or bandage treatment from a separate random draw as it was deemed unethical to withhold treatment to these cows.

Digital photographs of all hooves enrolled in the trial were taken before treatment. To ensure that lesion severity did not differ between treatments, initial lesions sizes were determined following the live animal trial phase of the experiment by measuring the largest diameter of the lesion from the digital photographs obtained on the day of initial treatment (exam 0). Actual sizes were calculated based on measurement of a standard- ized identification tag placed below each hoof at the time of photography. Lesion sizes were then categorized into lesion scores (Hernandez and Shearer, 2000) from 0 to 2 , due to the potential for small inaccuracies resulting from measurement methodology. Lesion scores of 0 consisted of no visible lesion, lesion scores of 1 were lesions $\leq 2.5 \mathrm{~cm}$, and scores of 2 were lesions $>2.5 \mathrm{~cm}$ in diameter.

To assess healing rates, a convenience sample of cows was reexamined at 2 time points following treatment: exam 1 (3 to $7 \mathrm{~d}$ posttreatment) and exam 2 (8 to $12 \mathrm{~d}$ posttreatment). As bandages were removed before reexaminations, the observer (author J. H. Higginson Cutler) was blind to treatment during these exams. At these time points, hooves were examined while cows were restrained in a standing trimming chute. Lesions were classified as active, healing, or healed; digital photographs were taken and the location of lesions was recorded.

Recurrence rates were determined throughout the 15-mo study period. A case was defined as recurred if digital dermatitis was present in the same location on the hoof at or before the following trim (usually a period of 3 to $4 \mathrm{mo}$ ). Farm veterinarians examined all lame cows identified by farm staff and any digital dermatitis lesions were classified by stage of healing, as well as having digital photographs taken and having anatomic locations recorded by the veterinarian. Farm staff had been trained to identify lame cows as part of their employment training.

Pressure algometry (Pain Test FPX 50 Algometer; Wagner Instruments, Greenwich, CT) was applied to each lesion site to quantify pain at each hoof exam. The mechanical nociceptive threshold (kilograms of force causing withdrawal of the limb from the algometer) was measured while cows were restrained in a chute with legs fastened with integrated restraints for hoof exams. The algometer tip, a 1-cm diameter flat rubber end, was pressed against the lesion with steadily increasing pressure until the nociceptive threshold was reached, as determined by leg withdrawal or avoidance response. A response of $0 \mathrm{~kg}$ was recorded when withdrawal occurred once the algometer touched the lesion. Algometer measurements were recorded in kilograms of force, and single measurements were obtained for each lesion to avoid irritating the lesion. The algometer measurements were censored at $25 \mathrm{~kg}$, the maximum value at which the device could record pressure.

\section{Statistical Analysis}

Statistical analysis was completed using SAS (SAS 9.2; SAS Institute Inc., Cary, NC). Descriptive statistics were compiled by examining means and frequencies to 
provide information on the characteristics of animals assigned to each treatment group and lesion information. To determine if the initial lesion scores differed among the 3 treatments, 1-way ANOVA (PROC NPAR1WAY) was used. Different models were then run to assess the effectiveness of treatments on lesion healing, recurrence rates, and relationship between pain and lesion healing. Parity, DIM, and lesion size were offered as covariates into all models, as well as the variables of interest: treatment, exam, and trim, with hoof as the statistical unit. Variables potentially associated with the outcome in univariable analysis with $P<0.2$ were included in the final models and then removed through backward elimination. A $P<0.05$ was considered significant in final models. Model fit was assessed through the examination of residuals.

To determine if healing rates differed among the treatment groups, a generalized linear mixed model (PROC GLIMMIX) with a binomial distribution (lesion healed or unhealed) was used. Lesions classified as both active and healing were grouped as unhealed. Random effects of cow and cow by treatment were included. Additionally, the hoof on which the lesion was located was treated as a repeated measure within cow and treatment.

To ascertain if the recurrence rate differed between the 2 treatment groups, a separate generalized linear mixed model with a logit link was used. For recurrence data (binomial distribution recurred or did not recur), only the paste and bandage treatments were analyzed, as those lesions assigned to the negative control group that were still active following collection of healing data may have been treated if deemed necessary. The cow was treated as a random effect and treatment group as a repeated measure to account for variance in covariance parameters.
Finally, to determine pain associated with active, healing, or healed cases of digital dermatitis regression analysis of survival data was used (PROC PHREG) due to the presence of censored data. Data from all treatment groups were included in analysis. The amount of pressure exerted at the lesion site before a withdrawal (in $\mathrm{kg}$ ) was used as an outcome, with $25 \mathrm{~kg}$ as a censored value. Repeated measures on individual animals were accounted for using robust sandwich variance estimates aggregated over cow, which allows for estimates of covariance matrix.

\section{RESULTS}

The characteristics of the animals and lesions assigned to each of the treatment groups are shown in Table 1 . Lesions were predominantly found in the hind limbs of cows, with 41.1 and $46.3 \%$ of lesions in the left hind and right hind limbs, respectively. Fewer lesions were observed in the front limbs, with $6.5 \%$ on the left and $6.1 \%$ on the right limbs. The majority of the lesions were located in zone 10 (Figure 1), accounting for $73.2 \%$ of all active lesions entered into the trial. Zone 0 contained $15.5 \%$ of the lesions, and $10.8 \%$ of lesions were in zone 6 . The remaining lesions $(0.5 \%)$ were observed in zone 4 . Whereas zones 4 and 6 normally comprise horn, horn deterioration and proliferation of digital dermatitis lesions was observed in these areas.

Lesion sizes were successfully measured from photographs for 157 of 219 hooves entered into the trial (exam 0); a proportion was not calculated due to poor quality of the photographs. Mean lesion scores are shown in Table 1.

Only some cows were accessible for examination following treatment due to their use in other research projects and the number of hooves examined can be

Table 1. Characteristics of Holstein cows assigned to paste, bandage, and negative control treatments in a randomized clinical trial examining tetracycline hydrochloride paste treatment efficacy for digital dermatitis ${ }^{1}$

\begin{tabular}{|c|c|c|c|c|}
\hline Item & \multicolumn{3}{|c|}{ Treatment } & $P$-value \\
\hline Parity $($ mean $\pm \mathrm{SD})$ & $1.8 \pm 0.14$ & $1.6 \pm 0.12$ & $1.9 \pm 0.14$ & 0.32 \\
\hline DIM (mean $\pm \mathrm{SD})$ & $188.1 \pm 13.0$ & $169.6 \pm 13.0$ & $158.9 \pm 13.7$ & 0.28 \\
\hline Nociceptive threshold ( $\mathrm{kg}$ of force; mean $\pm \mathrm{SD}$ ) & $10.0 \pm 10.5$ & $10.9 \pm 11.0$ & $11.2 \pm 10.3$ & 0.44 \\
\hline Exam $1(\mathrm{~d} 3-7)$ & 25 & 27 & 18 & \\
\hline$\%$ healed & 22.2 & 42.9 & 14.3 & \\
\hline Exam 2 (d 8-12) & 22 & 22 & 15 & \\
\hline$\%$ healed & 47.4 & 57.1 & 0 & \\
\hline Recurred lesions (\%) & 26.6 & 17.1 & - & 0.43 \\
\hline
\end{tabular}

\footnotetext{
${ }^{1}$ Exam days listed are relative to day of treatment.
} 


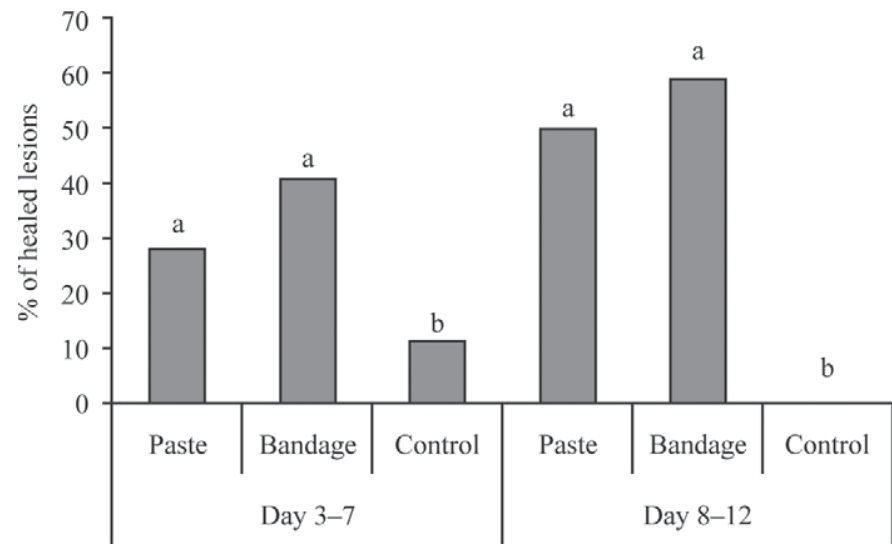

Figure 2. Percentage of observed lesions that were healed for the 3 different treatments (paste, bandage, and negative control) by exam time periods: exam 1 (d 5 to 7 of the trial) and exam 2 (d 8 to 12 of the trial). Columns containing different letters ( $\mathrm{a}$ and $\mathrm{b}$ ) are significantly different $(P<0.05)$.

found in Table 1. The number of healed lesions differed by the type of treatment $(P=0.0015$; Figure 2$)$. No difference existed between the odds of healing in the paste and bandage treatments (odds ratio $=0.299$, CI: $0.087-1.032, P=0.06$ ). The odds of finding a healed digital dermatitis lesion following assignment to the negative control group was 0.012 times the odds of having a healed lesion following bandage treatment (CI: $0.001-0.108, P=0.0005)$. Additionally, a difference was observed in the number of healed lesions among exam periods $(P=0.03$; Figure 2$)$. Lesions were more likely to be healed on $\mathrm{d} 8$ to 12 posttreatment compared with d 3 to $7(P=0.03)$. Trim date did not alter the odds of healing $(P=0.15)$.

Overall, 33 lesions recurred following treatment with either the paste or bandage treatments. Seventeen recurrences followed the June 2009 trim, 9 followed the September 2009 trim, 3 followed the January 2010 trim, and 4 followed the May 2010 trim. Twenty-one cows had been assigned to the paste group and 12 assigned to the bandage group. No difference was observed in the recurrence rates between the 2 treatment types $(P$ $=0.42)$. All other variables did not alter recurrence $(P$ $>0.05)$.

Algometer measurements were significantly different between lesions that were classified as active, healing, or healed $(P<0.0001 ;$ Figure 3$)$. The mean $( \pm \mathrm{SD})$ algometer measurement for lesions that were healed was $25.0( \pm 0) \mathrm{kg}$, the value at which measurements were censored. Lesions classified as healing had a mean of $12.84( \pm 10.49) \mathrm{kg}$ of force applied and active lesions had a mean of $7.90( \pm 9.45) \mathrm{kg}$ of force. Algometer measurements at exam 0 of active lesions varied greatly, as shown in Figure 4; however, for $50 \%$ of hooves tested, withdrawal responses were provoked at $3 \mathrm{~kg}$ of force or

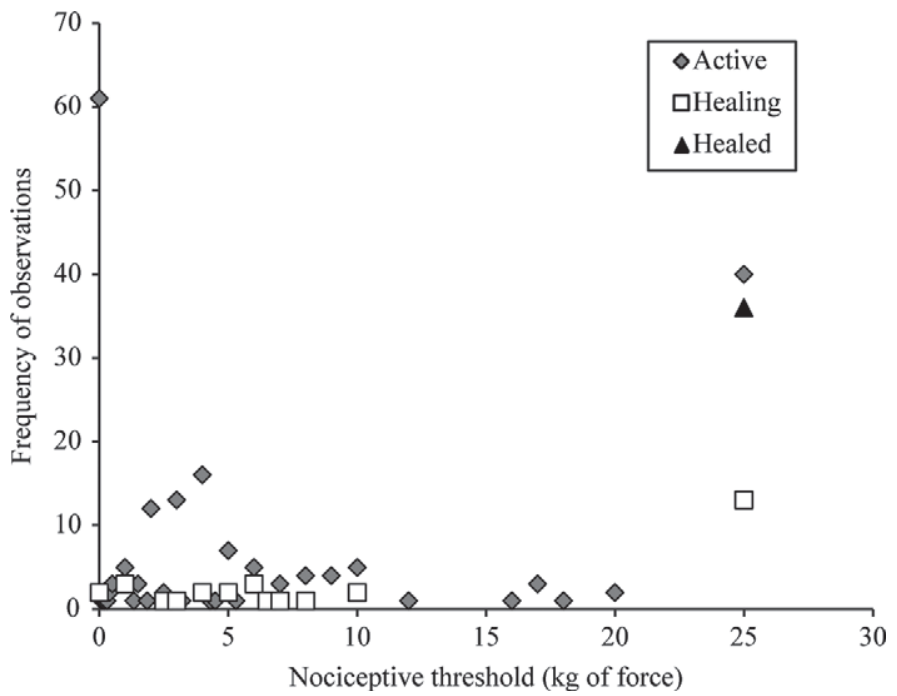

Figure 3. Frequency of observation in which cows responded at different nociceptive thresholds ( $\mathrm{kg}$ of force), applied by an algometer to digital dermatitis lesions, by healing status in a randomized clinical trial assessing the use of tetracycline hydrochloride paste treatment for digital dermatitis. Algometer measurements were made over 3 exam time periods: exam 0 (day of treatment assignment), exam 1 (d 5 to 7 of the trial), and exam 2 (d 8 to 12 of the trial).

less. Contrast statements demonstrated that all 3-way comparisons between classifications were significantly different $(P<0.001)$.

\section{DISCUSSION}

Infectious disease cure is normally defined by an absence of the causative agent. Debate still exists as to the identity of the causative agent of digital dermatitis, although the majority of experts agree that spirochetes are involved (Klitgaard et al., 2008; Evans et al., 2009).

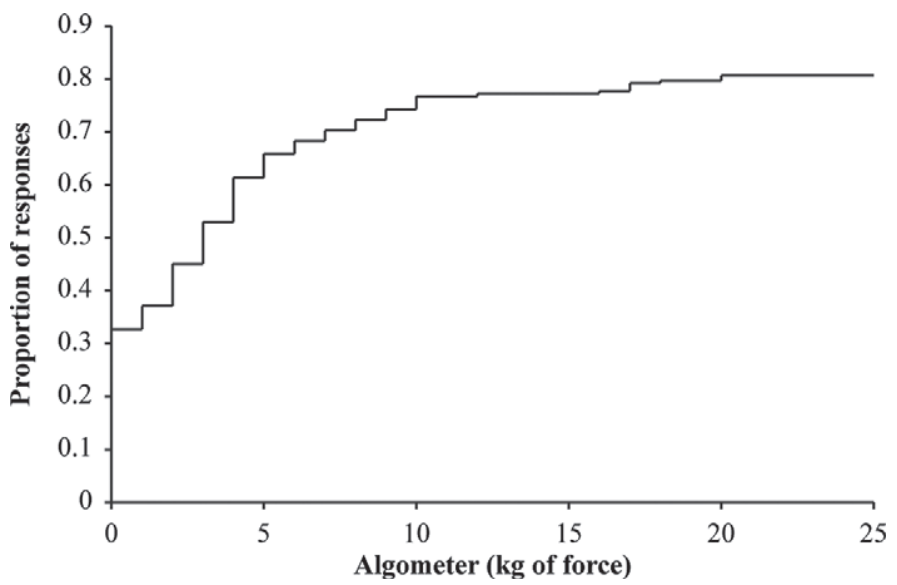

Figure 4. Proportion of responses by nociceptive threshold ( $\mathrm{kg}$ of force causing withdrawal of limb from the algometer) of active digital dermatitis lesions at exam 0 (day of treatment assignment). 
Therefore, the term healing rate was used in this paper and was based on visual observation of an absence of an active lesion.

Our results demonstrate that treatment of digital dermatitis with a tetracycline hydrochloride paste is as therapeutically effective as application of tetracycline hydrochloride held in place with a bandage for $2 \mathrm{~d}$ and would eliminate the necessity of bandage removal. Healing rates of digital dermatitis were not significantly different between these 2 treatments. Approximately $30 \%$ of lesions were examined at the 2 following exam periods due to availability of animals. At 8 to $12 \mathrm{~d}$ posttreatment, lesions were healed in 50 and $59 \%$ of cases for the paste and bandage treatments, respectively. These healing rates were numerically slightly lower than those previously published. Following topical application of an oxytetracycline solution, lesions were still visible in 4 of 11 cows 14 d after treatment (Hernandez et al., 1999). In another study using oxytetracycline bandage application, which was repeated after $5 \mathrm{~d}, 87 \%$ of hooves were considered cured $32 \mathrm{~d}$ after the initial treatment (Manske et al., 2002b). However, both studies followed lesion healing for a longer period of time than the maximum 12-d posttreatment in this study; therefore, it is not surprising that healing rates were higher.

The use of antibiotics in treatment in the dairy industry has been decreasing due to concerns about antibiotic resistance. For this reason, a convenience sample of 19 cows assigned to both the bandage and paste groups had composite milk samples taken throughout the trial period and no tetracycline hydrochloride residues above $50 \mu \mathrm{g} / \mathrm{kg}$ were found following treatment (Cramer et al., 2010).

Some of the lesions in the negative control group appeared to have healed during the 3 - to 7 -d posttreatment exam; however, all cows examined in the negative control group had active lesions at exam 2 (d 8 to 12). Digital dermatitis lesions have been shown to heal slowly on their own without any treatment over a period of 7 to $10 \mathrm{~d}$ (Blowey and Sharp, 1988). It is possible that these animals became reinfected between the 2 exam time periods, which may not have been observed in the other 2 treatments due to residual local antibiotic effect. Additionally, digital dermatitis lesions have been shown to frequently transition between disease states (Nielsen et al., 2012) perhaps due to the multiple symbiotic Treponema spirochete species (Klitgaard et al., 2008). This pattern was not observed in the 2 tetracycline hydrochloride treatment groups, with a greater number of healed lesions found during exam 2 (d 8 to 12 ) than exam 1 (d 3 to 7 ).

Digital dermatitis treatment to prevent recurrence of disease is limited by the ability of the product to reach the location of infection. Some treatments may only function to prevent recurrence of superficial digital dermatitis lesions, whereas others may reach deeper treponemes to prevent recurrence (Mumba et al., 1999; Nordhoff et al., 2008). This was likely a factor in the lack of difference in recurrence observed between treatments. Few studies have examined recurrence of digital dermatitis. One trial, in which 742 cows were observed over a 10 - to 12 -wk period, revealed a mean of $1 \%$ recurrence during that time period (Nielsen et al., 2012), a much lower percentage than the 17 to $26 \%$ observed over the same approximate time in the current trial. Another trial found that $33 \%$ of cows had recurrence of digital dermatitis over 7 to $12 \mathrm{wk}$ following use of a variety of antibiotic and nonantibiotic treatments (Read and Walker, 1998). Although lesions may heal due to the treatments applied, any irritation in the skin could allow for new infections of digital dermatitis. A posthoc power calculation, however, determined a need for 568 animals per group to determine a significant difference between digital dermatitis recurrence in the 2 treatments. The current study measured clinical cure and did not examine presence or absence of bacteria. Whereas recurrence at the following trim was assessed through presence of a digital dermatitis lesion in the same location, recurrence between trims was identified if a cow became lame and digital dermatitis was identified at the time of lameness exam. Recurrence of digital dermatitis between trims that did not lead to lameness would not have been accounted for in this data and lead to missing recurrence; however, due to successful randomization, it is assumed that this would not bias results between treatments.

Group mean lesion scores were calculated to ensure a similar severity of lesions in the different treatment groups. Lesions in the negative control group could have been less severe due to treatment assignment, as severe cases of digital dermatitis causing lameness were assigned to treatment from a separate randomization chart due to ethical concerns of animal suffering, which could have biased results. Although a small numerical difference existed, no statistically significant difference was found among treatment groups.

To more accurately and empirically quantify a pain response, a pressure algometer was used to measure the nociceptive threshold at the lesion site in this randomized clinical trial. Pressure algometry has been used to quantify pain with calf disbudding by measuring mechanical nociceptive thresholds (Heinrich et al., 2010). In that research, nociceptive thresholds of calves at the site of disbudding increased (disbudding was less painful to calves) with provision of a nonsteroidal antiinflammatory drug compared with a placebo, supporting the assumption that pressure algometry is measuring pain. Nociceptive thresholds have been successfully used to 
measure pain in lame sheep (Ley et al., 1995) and cattle (Whay et al., 1998) when measuring hyperalgesia at locations proximal to the lesion of interest on the limb, using force with a blunt pin. Flocks of sheep with high levels of lameness due to foot rot had lower nociceptive thresholds (or increased response to painful stimulus) than matched sound controls (Ley et al., 1995). Sole ulcers and white line disease have also been associated with lower nociceptive thresholds in cattle on the day of treatments as well at $28 \mathrm{~d}$ later compared with sound cows, whereas cows with acute tissue infections, including digital dermatitis, were significantly different from sound cows on the day of treatment (Whay et al., 1998). Dyer et al. (2007) also used nociceptive threshold in dairy cattle with different lesions with the use of hoof testers equipped with a pressure gauge to determine claw pain and an algometer to measure integument pain, to determine a combined claw pain index. These methods combined indicated that claw pain increased in the lateral claws with increasing locomotion score, or increase in lameness, indicating more pain in lame cattle.

The current study used pressure algometry to evaluate nociceptive threshold at the site of digital dermatitis lesions at different stages of healing. Nociceptive threshold measurements with the algometer may not fully reflect the amount of pain the cow experiences from digital dermatitis due to their restraint in a trimming chute during examination. Restraint for claw trimming is believed to be stressful, causing an elevation in fecal cortisol metabolites (Pesenhofer et al., 2006). Stressful events can lead to stress-induced analgesia (briefly reviewed in Veissier et al., 2000), which would increase the nociceptive threshold response. In addition, physical restraint of the leg decreases the amount of movement the cow can perform, leading to a decrease in obvious leg withdrawal or avoidance response. Use of pressure algometry on areas that are less restrained, such as horn buds in the study of Heinrich et al. (2010), allow for more movement and a potentially lower nociceptive threshold. Therefore, pain responses for the 2 studies would not be comparable.

Healed lesions did not appear to be painful, as all healed lesions having an algometer measurement reached the censored value of $25 \mathrm{~kg}$. Cows responded to less application of force with the algometer, or had a lower nociceptive threshold, when lesions were active compared with healing or healed lesions, indicating that these lesions were painful. These results support the use of pressure algometry as a method for evaluation pain in these lesions. However, it was surprising that pressure algometry applied to $19 \%$ of active lesions failed to provoke a withdrawal or avoidance response.
In addition to restraint causing a lack of response, it is possible that different stages of disease progression cause variable amounts of pain. Future studies using a lesion severity scale in addition to algometer measurements could provide more information about pain during digital dermatitis infection. More frequent repeated measures might also provide further information about healing. Significant pain responses were also observed during the healing stages, with cows twice as sensitive to pressure on the healing lesion site than when healed, suggesting a prolonged duration of significant pain.

Due to the pain experienced by cows with active and healing digital dermatitis lesions, treatment options should include pain management interventions. The algometer is a tool that could be used to determine the amount of pain experienced by individual animals infected with digital dermatitis and determine effectiveness of intervention strategies. Further research into pain mitigation and management changes that could assist the convalescent cow are necessary for appropriate care.

\section{CONCLUSIONS}

The use of a tetracycline paste treatment was as effective as a bandage containing tetracycline hydrochloride powder in terms of both healing rates and digital dermatitis recurrence. This indicates that paste treatments could be effectively used for the treatment of digital dermatitis and would eliminate the need for bandage removal, providing benefits for animal welfare, health, and convenience. Digital dermatitis lesions can be painful during both active and healing stages, suggesting the need for treatment and husbandry interventions for pain mitigation.

\section{ACKNOWLEDGMENTS}

The authors thank Laura Wright and the staff at the Elora Dairy Research Centre (Elora, ON, Canada) for technical assistance with the trial, Rob Swackhammer (Swackhammer Veterinary Services, Rockwood, ON, Canada) and Lisa Baarda (Cramer Mobile Bovine Veterinary Services, Stratford, ON, Canada) for assistance with hoof examinations, and William Sears at the University of Guelph (Guelph, ON, Canada) for assistance with statistical analysis. Additionally, Chuck Guard (Cornell University, Ithaca, NY) is thanked for promoting use of a paste formula for digital dermatitis treatment. Funding for this project was provided by the National Sciences and Engineering Research Council of Canada (Ottawa, ON, Canada) and Dairy Farmers of Ontario (Mississauga, ON, Canada). 


\section{REFERENCES}

Anderson, D. M., and R. A. S. White. 2000. Ischemic bandage injuries: A case series and review of the literature. Vet. Surg. 29:488-498.

Berry, S. L., D. H. Read, R. L. Walker, and T. R. Famula. 2010. Clinical, histologic, and bacteriologic findings in dairy cows with digital dermatitis (footwarts) one month after topical treatment with lincomycin hydrochloride or oxytetracycline hydrochloride. J. Am. Vet. Med. Assoc. 237:555-560.

Blowey, R. W., and M. W. Sharp. 1988. Digital dermatitis in dairy cattle. Vet. Rec. 122:505-508.

Cheli, R., and C. M. Mortellaro. 1974. La dermatite digitale del bovino. Pages 208-213 in Proc. 8th Int. Conf. Dis. Cattle, Milan, Italy.

Cramer, G., J. Higginson, and R. Tenbergen. 2010. Lack of tetracycline residues with two different methods of topical oxytetracycline treatment for digital dermatitis. Ceptor Animal Health News 18:19.

Cramer, G., K. D. Lissemore, C. L. Guard, K. E. Leslie, and D. F. Kelton. 2008. Herd- and cow-level prevalence of foot lesions in Ontario dairy cattle. J. Dairy Sci. 91:3888-3895.

Dyer, R. M., N. K. Neerchal, U. Tasch, Y. Wu, P. Dyer, and P. G. Rajkondawar. 2007. Objective determination of claw pain and its relationship to limb locomotion score in dairy cattle. J. Dairy Sci. 90:4592-4602.

Evans, N. J., J. M. Brown, I. Demirkan, R. Birtles, C. A. Hart, and S. D. Carter. 2009. In vitro susceptibility of bovine digital dermatitis associated spirochaetes to antimicrobial agents. Vet. Microbiol. $136: 115-120$

Galindo, F., and D. M. Broom. 2002. The effects of lameness on social and individual behavior of dairy cows. J. Appl. Anim. Welf. Sci. $5: 193-201$

Heinrich, A., T. F. Duffield, K. D. Lissemore, and S. T. Millman. 2010. The effect of meloxicam on behavior and pain sensitivity of dairy calves following cautery dehorning with a local anesthetic. J. Dairy Sci. 93:2450-2457.

Hernandez, J., and J. K. Shearer. 2000. Efficacy of oxytetracycline for treatment of papillomatous digital dermatitis lesions on various anatomic locations in dairy cows. J. Am. Vet. Med. Assoc. $216: 1288-1290$.

Hernandez, J., J. K. Shearer, and J. B. Elliott. 1999. Comparison of topical application of oxytetracycline and four nonantibiotic solutions for treatment of papillomatous digital dermatitis in dairy cows. J. Am. Vet. Med. Assoc. 214:688-690.

Klitgaard, K., M. Boye, N. Capion, and T. K. Jensen. 2008. Evidence of multiple Treponema phylotypes involved in bovine digital dermatitis as shown by $16 \mathrm{~S}$ rRNA gene analysis and fluorescence in situ hybridization. J. Clin. Microbiol. 46:3012-3020.
Laven, R. A., and D. N. Logue. 2006. Treatment strategies for digital dermatitis for the UK. Vet. J. 171:79-88.

Laven, R. A., and M. J. Proven. 2000. Use of an antibiotic footbath in the treatment of bovine digital dermatitis. Vet. Rec. 147:503-506.

Ley, S. J., A. E. Waterman, and A. Livingston. 1995. A field study of the effect of lameness on mechanical nociceptive thresholds in sheep. Vet. Rec. 137:85-87.

Manske, T., J. Hultgren, and C. Bergsten. 2002a. The effect of claw trimming on the hoof health of Swedish dairy cattle. Prev. Vet. Med. 54:113-129.

Manske, T., J. Hultgren, and C. Bergsten. 2002b. Topical treatment of digital dermatitis associated with severe heel-horn erosion in a Swedish dairy herd. Prev. Vet. Med. 53:215-231.

Mumba, T., D. Döpfer, C. Kruitwagen, M. Dreher, W. Gaastra, and B. A. M. van der Zeijst. 1999. Detection of spirochetes by polymerase chain reaction and its relation to the course of digital dermatitis after local antibiotic treatment in dairy cattle. Zentralbl. Veterinärmed. B 46:117-126.

Nielsen, B. H., P. T. Thomsen, L. E. Green, and J. Kaler. 2012. A study of the dynamics of digital dermatitis in 742 lactating dairy cows. Prev. Vet. Med. 104:44-52.

Nordhoff, M., A. Moter, K. Schrank, and L. H. Wieler. 2008. High prevalence of treponemes in bovine digital dermatitis - A molecular epidemiology. Vet. Microbiol. 131:293-300.

Pesenhofer, G., R. Palme, R. M. Pesenhofer, and J. Kofler. 2006. Comparison of two methods of fixation during functional claw trimming - Walk-in crush versus tilt table - In dairy cows using faecal cortisol metabolite concentrations and daily milk yield as parameters. Vet. Med. Austria 93:288

Read, D. H., and R. L. Walker. 1998. Papillomatous digital dermatitis (footwarts) in California dairy cattle: Clinical and gross pathologic findings. J. Vet. Diagn. Invest. 10:67-76.

Shearer, J., D. Anderson, W. Ayars, E. Belknap, S. Berry, C. Guard, K. Hoblet, E. Hovingh, G. Kirksey, A. Langill, A. Mills, D. Miskimins, J. Osterstock, R. Price, D. Prigel, A. Roussel, S. van Amstel, S. R. Wallace, J. Wasson, N. Cook, E. F. Garrett, D. E. Hostetler, and L. Schugel. 2004. A record-keeping system for capture of lameness and foot-care information in cattle. Bovine Pract. 38:83-91.

Veissier, I., J. Rushen, D. Colwell, and A. M. de Passillé. 2000. A laserbased method for measuring thermal nociception of cattle. Appl. Anim. Behav. Sci. 66:289-304

Wells, S. J., L. P. Garber, and B. A. Wagner. 1999. Papillomatous digital dermatitis and associated risk factors in US dairy herds. Prev. Vet. Med. 38:11-24.

Whay, H. R., A. E. Waterman, A. J. Webster, and J. K. O'Brien. 1998 The influence of lesion type on the duration of hyperalgesia associated with hindlimb lameness in dairy cattle. Vet. J. 156:23-29. 